\title{
HOLOMORPHIC SECTIONAL CURVATURE OF QUASI-SYMMETRIC DOMAINS
}

\author{
R. ZELOW (LUNDQUIST)
}

\begin{abstract}
It is well known that the holomorphic sectional curvature of a bounded symmetric domain is bounded above by a negative constant. In this paper we show that this is true more generally for a quasi-symmetric Siegel domain, and the proof is based on a formula for the curvature from the author's thesis. The bounded homogeneous domains are, as is well known, biholomorphic to homogeneous Siegel domains and the bounded symmetric domains are biholomorphic to those quasi-symmetric (homogeneous) Siegel domains that satisfy a certain algebraic identity (which we do not need here).
\end{abstract}

In a recent paper [7] we have determined a formula for the holomorphic sectional curvature of an irreducible quasi-symmetric Siegel domain, and proved that it is $<0$. Here we refine this result and prove that the holomorphic sectional curvature is bounded above by a negative constant. We recall some standard facts for which [4] is a good reference, and we use the notation in [4] and [7].

Let $\Omega$ be an open convex homogeneous cone in $\mathbf{R}^{n}$, not containing any straight line, and assume $\Omega$ is self-dual with respect to an inner product $\langle$, $\rangle$ on $\mathbf{R}^{n}$, i.e.,

$$
\left\{y \in \mathbf{R}^{n} \mid\left\langle y, y^{\prime}\right\rangle>0 \forall y^{\prime} \in \bar{\Omega}-\{0\}\right\}=\Omega .
$$

Further let $F: \mathbf{C}^{m} \times \mathbf{C}^{m} \rightarrow \mathbf{C}^{n}$ be an $\Omega$-hermitian form, which is conjugate linear in the second variable,

$$
F\left(w_{1}, w_{2}\right)=\overline{F\left(w_{2}, w_{1}\right)}, \quad F(w, w) \in \bar{\Omega}, \quad F(w, w)=0 \Leftrightarrow w=0 .
$$

We then have the Siegel domain

$$
\mathscr{D}=\mathscr{D}(\Omega, F):=\left\{(z, u) \in \mathbf{C}^{n} \times \mathbf{C}^{m} \mid \operatorname{Im} z-F(u, u) \in \Omega\right\},
$$

which is biholomorphic to a bounded homogeneous domain. Let $e \in \Omega$ be the base point with the property that the stability subgroup of

$$
G(\Omega):=\{A \in \mathrm{Gl}(n, \mathbf{R}) \mid A \Omega=\Omega\}
$$

at $e$ is $K_{e}=\left\{A \in G(\Omega) \mid A^{\prime}=A^{-1}\right\}$, where ' is transpose with respect to $\langle$, (see [4]). Let $g(\Omega)=\mathfrak{f}_{e}+\mathfrak{p}_{e}$ be the Cartan decomposition of the Lie algebra $\mathrm{g}(\Omega)$ of $G(\Omega)$ at $e$. We then have the mappings $T$ and $R$ of Satake [4]:

\footnotetext{
Received by the editors June 6, 1978 and, in revised form, November 15, 1978.

AMS (MOS) subject classifications (1970). Primary 53C35; Secondary 32M15.

Key words and phrases. Bounded homogeneous domain, bounded symmetric domain, Bergman metric, quasi-symmetric Siegel domain, cone, tube domain.
} 


$$
\mathbf{R}^{n} \ni a \mapsto T_{a} \in \mathfrak{p}_{e} \subset \mathfrak{g}(\Omega) \subset \mathfrak{g l}(n, \mathbf{R})
$$

defined uniquely by $T_{a} e=a$. With the product $a_{1} \circ a_{2}=T_{a_{1}} a_{2}, \mathbf{R}^{n}$ becomes a formally real Jordan algebra. Further, for $a \in \mathbf{R}^{n}$ let $R_{a} \in \mathfrak{g l}(m, \mathbf{C})$ be defined by

$$
\left\langle a, F\left(v_{1}, v_{2}\right)\right\rangle=2\left\langle e, F\left(v_{1}, R_{a} v_{2}\right)\right\rangle .
$$

Then the following conditions are equivalent [4]:

(i) For $a \in \mathbf{R}^{n}$ we have

$$
T_{a} F\left(v_{1}, v_{2}\right)=F\left(R_{a} v_{1}, v_{2}\right)+F\left(v_{1}, R_{a} v_{a}\right) \quad \forall v_{1}, v_{2} \in \mathbf{C}^{m}
$$

(ii) For $a_{1}, a_{2} \in \mathbf{R}^{n}$ we have $R_{a_{1} \circ a_{2}}=R_{a_{1}} R_{a_{2}}+R_{a_{2}} R_{a_{2}}$.

If this holds, then $\mathscr{D}$ is said to be quasi-symmetric [4]. Every bounded symmetric domain is biholomorphic to such a domain $\mathscr{D}$. We assume that $\mathscr{D}$ is irreducible, i.e., not expressible as (biholomorphic to) a product of two bounded homogeneous domains, which is equivalent to $\Omega$ being irreducible, i.e. not expressible as a product of two cones [1].

We extend $\langle$,$\rangle , \circ$ and $R_{a}$ by linearity to elements in $\mathbf{C}^{n}$. Letting

$$
\begin{gathered}
\partial_{z}:=\left(\frac{\partial}{\partial z^{1}}, \ldots, \frac{\partial}{\partial z^{n}}\right), \quad \partial_{u}:=\left(\frac{\partial}{\partial u^{1}}, \ldots, \frac{\partial}{\partial u^{m}}\right), \\
a \cdot \partial_{z}:=a_{1} \frac{\partial}{\partial z^{1}}+\cdots+a_{n} \frac{\partial}{\partial z^{n}},
\end{gathered}
$$

etc., and letting $\sigma=(i e, 0)$ be the base point of $\mathscr{D}$, we have [7]:

If $\langle,\rangle_{\sigma}$ is the Bergman (bilinear) metric of $\mathscr{D}$ at $\sigma$, then the holomorphic sectional curvature (with respect to the Bergman metric) determined by $Z=a \cdot \partial_{z}+b \cdot \partial_{u}$ with $\langle Z, \bar{Z}\rangle_{\sigma}=1$ is

$$
\begin{aligned}
K(Z)=-C\{ & \frac{1}{4}[2\langle a \circ \bar{a}, a \circ \bar{a}\rangle-\langle a \circ a, \bar{a} \circ \bar{a}\rangle] \\
& \left.+8\left\langle e, F\left(R_{\bar{a}} b, R_{\bar{a}} b\right)\right\rangle+4\langle F(b, b), F(b, b)\rangle\right\}
\end{aligned}
$$

where $C$ is a positive constant.

We can now state:

THEOREM. If $\mathscr{D}=\mathscr{D}(\Omega, F)$ is a quasi-symmetric domain, then the holomorphic sectional curvature is bounded above by a negative constant.

REMARKS. For bounded symmetric domains this is well known. To prove the theorem we can assume that $\Omega$ is irreducible. Then either $\Omega=\mathscr{P}_{r}(\mathbf{F})$, positive symmetric matrices of size $r \times r$ with coefficients in $F=R, C, H$ (real numbers, complex numbers, quaternions), or

$$
\Omega=S_{n}=\left\{x \in \mathbf{R}^{n} \mid x_{1} x_{2}-x_{3}^{2}-\cdots-x_{n}^{2}>0, x_{1}>0\right\},
$$

or $\Omega$ is the exceptional cone $\mathscr{P}_{3}$ (Cay). (See [4], [6], [7].) For this last cone it is known (Satake) that $\mathscr{D}$ is the tube domain with this cone, and hence $\mathscr{D}$ is symmetric. So the theorem holds for this domain. The above curvature formula was proved in [7] for the cones $\mathscr{P}_{r}(F), S_{n}$. So now we have to prove 
the theorem for these cones. Since the holomorphic sectional curvature of a complex submanifold does not exceed that of the ambient complex manifold, the result is also known for those quasi-symmetric domains that occur as fibers of "boundary-fibrations" of bounded symmetric domains over their boundary components ([3], [4], [8]). However, not all quasi-symmetric domains occur as such fibers.

Proof of The TheOREM. If one writes $a=\alpha+i \beta$ with $\alpha, \beta \in \mathbf{R}^{n}$, then

$$
\begin{aligned}
2\langle a \circ \bar{a}, a \circ \bar{a}\rangle-\langle a \circ a, \bar{a} \circ \bar{a}\rangle \\
\quad=4\langle\alpha \circ \alpha, \beta \circ \beta\rangle+\langle(\alpha+\beta) \circ(\alpha+\beta),(\alpha-\beta) \circ(\alpha-\beta)\rangle .
\end{aligned}
$$

Both terms on the right-hand side are $>0$, since (see [4]) $x \circ x \in \bar{\Omega}$ for any $x \in \mathbf{R}^{n}$ and since $\Omega$ is self-dual. Also the terms $\left\langle e, F\left(R_{\bar{a}} b, R_{\bar{a}} b\right)\right\rangle$ and $\langle F(b, b), F(b, b)\rangle$ in the curvature formula are $>0$; the first one since $e$, $F\left(R_{\bar{a}} b, R_{\bar{a}} b\right) \in \bar{\Omega}$ and $\Omega$ is self-dual, the second one since $F(b, b) \in \bar{\Omega} \subset \mathbf{R}^{n}$. So $K(Z)<0$. If it now happens that $K(Z)=0$, then all three terms in the formula vanish. In particular $F(b, b)=0$, and so $b=0$. Hence $a \neq 0$. However, this is exactly the situation we would have (with another constant $C$ ) if we tried to see if

$$
K(Z)=-C[2\langle a \circ \bar{a}, a \circ \bar{a}\rangle-\langle a \circ a, \bar{a} \circ \bar{a}\rangle]
$$

could vanish for $Z=a \cdot \partial_{z}$ with $\langle Z, \bar{Z}\rangle_{\sigma}=1$ in the case of the tube domain $\mathscr{D}=\mathbf{R}^{n}+i \Omega$, which is symmetric. Since the theorem is known in this case, we must have $2\langle a \circ \bar{a}, a \circ \bar{a}\rangle-\langle a \circ a, \bar{a} \circ \bar{a}\rangle\rangle 0$, contradicting the above statement that this expression vanishes. Hence $K(Z)<0$ for $Z \neq 0$ also in the case of an irreducible quasi-symmetric domain. By compactness of $\left\{Z=a \cdot \partial_{z}+b \cdot \partial_{u} \mid\langle Z, \bar{Z}\rangle_{\sigma}=1\right\}$ the theorem follows. Q.E.D.

It is also possible to give a direct, computational proof of the theorem.

\section{BIBLIOGRAPHY}

1. S. Kaneyuki, On the automorphism groups of homogeneous bounded domains, J. Fac. Sci. Univ. Tokyo 14 (1967), 87-130.

2. S. Murakami, On automorphisms of Siegel domains, Lecture Notes in Math., vol. 286, Springer-Verlag, Berlin and New York, 1972.

3. I. I. Pjateckii-Sapiro, Automorphic functions and the geometry of classical domains, Gordon and Breach, New York, 1969.

4. I. Satake, On the classification of quasi-symmetric domains, Nagoya Math. J. 62 (1976), 1-12.

5. __ Forthcoming book about algebraic structures on symmetric domains (to appear).

6. M. Takeuchi, Homogeneous Siegel domains, Publications of the Study Group of Geometry, vol. 7, Institute of Mathematics, Yoshida College, Kyoto Univ., Kyoto, 1973.

7. R. Zelow (Lundquist), Curvature of quasi-symmetric domains, J. Differential Geometry (to appear).

8. __ On the geometry of some Siegel domains, Nagoya Math. J. 73 (1979), 175-195.

Department of Mathematics, University of California, Berkeley, Californi 94720

Current address: Sverre Iversensvei 15, Oslo 9, Norway 